\title{
TECNOLOGÍAS DEL HÁBITAT URBANO E INFRAESTRUCTURA EN LA POLÍTICA DE GESTIÓN DE RIESGOS DE PARAGUAY
}

\author{
Eje 4: Hábitat e infraestructura
}

Flores López Moreira, Ana Raquel

\begin{abstract}
Área de Tecnología y Hábitat, Facultad de Arquitectura Diseño y Arte, Universidad Nacional de Asunción, San Lorenzo, Paraguay, www,arq.una.py, 021595 585558, anaraflo@hotmail.com
\end{abstract}

\section{RESUMEN}

La creciente concentración de población en las ciudades, en el marco de una débil planificación y procesos de urbanización rápida, incrementan la exposición de las poblaciones y sus bienes a diversas amenazas antrópicas o naturales ${ }^{1}$, al igual que, la vulnerabilidad ante estas, tal como se ha visto en diversos estudios y también en la investigación "FADA UNA, actor facilitador de la gestión urbana interinstitucional, para la llegada articulada de las Políticas Sociales a los Asentamientos Precarios Urbanos en situación de Riesgo"2.

Siendo la cuestión de los Riesgos Urbanos y su gestión, un tema de desarrollo relativamente reciente, por imperio de los acontecimientos, su tratamiento se ha vuelto prioritario para los países, al influjo del liderazgo de la Organización de las Naciones Unidas- ONU y otros organismos internacionales, que se ocupan de la misma, aportando conocimiento y orientaciones para su aplicación en las Políticas Públicas relacionadas al Hábitat construido. Entre las más recientes, el Marco de Hyogo y Sendai para la reducción del Riesgo de desastres, la Agenda 2030 para el Desarrollo Sostenible o la Nueva Agenda Urbana H III, resultantes del análisis de la situación global, orientan las acciones y generan compromisos de los países signatarios de las resoluciones tomadas en sendas reuniones de estadistas. En este contexto, y en la línea de investigación de la autora, la temática propuesta para el X CRETA constituye una oportunidad para revisar la Política de Gestión de Riesgos en Paraguay, cotejándola con las disposiciones de los instrumentos antes mencionados, a efectos de evidenciar los aspectos ausentes o de débil tratamiento en la misma, respecto de las Tecnologías del Hábitat e infraestructura. Tecnologías de Gestión, aportando dicha evidencia a sus lineamientos correspondientes.

\section{PALABRAS CLAVES: TECNOLOGÍAS DEL HÁBITAT E INFRAESTRUCTURA - POLÍTICA - GESTIÓN DE RIESGOS - LINEAMIENTOS}

\footnotetext{
${ }^{1}$ Inundaciones súbitas debidas a puntuales precipitaciones pluviales intensas o cíclicas como las que afectan diversas zonas del Paraguay desde el año 2012 y a los países vecinos que comparten ríos internacionales.

2 Desarrollada por la autora en el marco del Proyecto de Docente investigador de Dedicación Completa- DIDCom 20142016.
} 


\section{INTRODUCCIÓN}

En relación con el Eje Hábitat e Infraestructura. Tecnologías de gestión, que interesa al X CRETA, éste trabajo se apoya en los conceptos de algunos autores, como Arango Escobar (1986), para quien Tecnologías del hábitat constituye el "conjunto de decisiones y acciones tecnológicas orientadas a la producción, consolidación y preservación del hábitat" (Arango, 1986: Pág.1). Distingue así mismo, los "Componentes Físicos de Tecnología" asociados a hechos físicos (materiales, fábricas, productos, obras de infraestructura, etc.) y los "Componentes Lógicos de la tecnología" (asociados a procesos tecnológicos intangibles: conocimientos, técnicas, experiencias, procedimientos, capacitación, educación, aspectos socioculturales, antropológicos, etc.), que influyen significativamente en el desarrollo tecnológico" (Arango, 1986: Pág. 4, 5).

Por su parte González Escobar (2001), señala que, a partir de la segunda mitad del siglo XIX, la tecnología, pasó a verse "... no sólo en términos instrumentales, de herramientas, equipos, aparatos y otros productos materiales físicos, sino también como procesos de producción sistemáticos de hechos intangibles" (...), tales como la "...producción de condiciones psicológicas, sociales y políticas como parte de cualquier cambio técnico significativo" (González Escobar,2001: Pág. 19, 20). Mencionando también la clasificación de las tecnologías en Duras y Blandas, González Escobar (2001) refiriere las últimas, a organizaciones empresariales o institucionales, afirmando que "...las tecnologías dejaron de ser hechos sólo tangibles para asumir los intangibles, y en ambos casos son elementos fundamentales constitutivos de la integralidad del hábitat del hombre (...) con “...diversas formas de habitabilidad y de producción de técnicas, que de manera permanente configuran y transforman sus hábitos y las dinámicas y formas de organización de su habitar" (...) "...el éxito o el fracaso de un hábitat, parte de la capacidad de respuesta a la necesidad de habitar (...) en la historia se ha visto como muchas sociedades han perecido por las catástrofes ocurridas de malas aplicaciones tecnológicas sobre su hábitat", por lo que se requiere "...un equilibrio entre los materiales físicos y culturales, y su capacidad de articulación y apropiación armónica de los entornos de soporte (González Escobar,2001: Pág. 23, 25, 26).

Ciertamente, es posible observar en los últimos tiempos la intensificación de los problemas urbanos no resueltos, que se profundizan con los desplazamientos poblacionales (rural- urbano y/o internacionales, derivados generalmente de políticas económicas y de uso territorial agropecuario extensivo) a las ciudades, las que, con débiles soportes de planificación urbana y capacidad instalada, encuentran ingentes dificultades para enfrentar la urbanización rápida en condiciones adecuadas. La manifestación de esta condición se aprecia fundamentalmente en las infraestructuras precarizadas, creando al mismo tiempo condiciones de exposición cada vez mayor, de la población urbana a los efectos de los fenómenos naturales o antrópicos generadores de riesgos y desastres.

Zoido et al (2013), conciben la infraestructura "...como el conjunto de espacios, edificaciones y conducciones existentes en los núcleos urbanos, destinados a abastecer, eliminar residuos y comunicar o relacionar las distintas partes de la ciudad entre sí y a estas con el conjunto del territorio en que se localiza (Zoido et al,2013: Pág. 204). El concepto incluye a las obras subterráneas (redes de gas, agua potable o alcantarillado) y también a las obras superficiales (carreteras, vías férreas, instalaciones portuarias y aeroportuarias, redes de distribución de energía eléctrica, telefónicas y TV cable), consideradas mínimas para urbanizar un determinado suelo, y por 
extensión de la obra pública en general, a los edificios institucionales, salud, educación y viviendas proveídas por el Estado.

Más recientemente, Hábitat III, respecto de la infraestructura y servicios básicos, refiere al suministro de agua potable, saneamiento, gestión de residuos, el bienestar social, los servicios de transporte y comunicaciones, energía, servicios de salud y de emergencia, escuelas, seguridad pública, y la gestión de los espacios abiertos, y se destaca, que, durante la última década, el concepto se ha extendido hasta concebirse como un sistema que incluye:

- redes de activos (estructuras e instalaciones físicas y los vínculos internos entre estas).

- el conocimiento (recursos humanos que participan en la planificación, diseño, construcción y operación; los marcos legales y regulatorios; la política, los planes, la legislación, reglamentaciones, códigos, planes de desarrollo estratégico nacionales, regionales o locales) que orienta las inversiones, la toma de decisiones y priorización relacionados a los sistemas de infraestructura, y

- las instituciones componentes del sistema y sus capacidades, que garantizan la viabilidad financiera, la regulación, la planificación, la gestión y operación de la infraestructura urbana (Hábitat III, 2015:)

Ésta ponencia se plantea en el contexto conceptual precedentemente expuesto y su desarrollo se dirige a la revisión de las normas internacionales (MAH; SENDAI; ODS 2030; NAU) a los efectos de detectar los aspectos relevantes de las mismas, concernientes a la Tecnología del Hábitat e Infraestructura y a la consideración de éstos aspectos en relación con la Política de Gestión de Riesgos- PGR del Paraguay, como instrumento y Tecnología de Gestión del Hábitat, para aportar a los lineamientos correspondientes de dicho instrumento, aquellos aspectos que no estén presentes o que deban ser fortalecidos, a la luz de los riesgos y eventos de desastre cuyos efectos se han intensificado en el país.

\section{DESARROLLO}

\subsection{Normas Internacionales consideradas.}

En un contexto de pérdidas derivadas de las diversas situaciones de desastres ocurridas en el mundo, que afectaron más de 200.000.000 de personas en las 2 últimas décadas anteriores al 2005, así como "...la supervivencia, la dignidad y los medios de vida de los seres humanos, en particular los pobres (...) y también de la creciente vulnerabilidad generada "...por la evolución de las condiciones demográficas, tecnológicas y socioeconómicas, la urbanización sin plan, el desarrollo en zonas de alto riesgo, el subdesarrollo, la degradación del medio ambiente, el cambio climático, las amenazas geológicas, la competencia por los recursos escasos y el impacto de epidemias como la del VIH/SIDA..." (MAH, 2005: Pág. 1) se realizó la Conferencia Mundial sobre la Reducción de los Desastres celebrada en Kobe, Hyogo (Japón- 18 al 22 de enero de 2005), de la que derivó el Marco de Acción de Hyogo- MAH 2005-2015, como instrumento estratégico y sistemático de reducción de la vulnerabilidad a las amenazas/peligros y los riesgos que éstos conllevan, que destaca la necesidad de aumentar la resiliencia de las naciones y las comunidades ante los desastres.

Respecto de la Gestión de Riesgos- GR, en la oportunidad fueron señaladas deficiencias en la Gobernanza: marcos institucionales, jurídicos y normativos; en la Identificación, evaluación y vigilancia de los riesgos y alerta temprana; en la Gestión de los conocimientos y educación; en la 
Reducción de los factores de riesgo subyacentes; y en la Preparación para una respuesta eficaz y una recuperación efectiva, lo cual dio origen a $\mathbf{5}$ Acciones prioritarias, para ser consideradas en los Planes y Programas de los países signatarios del mundo, a nivel nacional y local, entre los años 2005 y 2015. Ellas son:

1. Velar por que la reducción de los riesgos de desastre constituya una prioridad nacional y local dotada de una sólida base institucional de aplicación; 2. Identificar, evaluar y vigilar los riesgos de desastres y potenciar la alerta temprana; 3. Utilizar los conocimientos, las innovaciones y la educación para crear una cultura de seguridad y de resiliencia a todo nivel; 4 . Reducir los factores de riesgo subyacentes; 5 . Fortalecer la preparación para casos de desastre a fin de lograr una respuesta eficaz.

Sucedió a esta herramienta, el Marco de Acción de Sendai - SENDAI 2015-2030, el que, a su vez, plantea 4 Acciones prioritarias: 1. Comprender el riesgo de desastres; 2. Fortalecer la Gobernanza del riesgo de desastres para gestionar dicho riesgo; 3. Invertir en la reducción del riesgo de desastres para la resiliencia; 4 . Aumentar la preparación para casos de desastres a fin de dar una respuesta eficaz y "reconstruir mejor" en los ámbitos de la recuperación, la rehabilitación y la reconstrucción.

De los 17 Objetivos del Desarrollo Sostenible - ODS 2030, se han revisado particularmente los siguientes: 6. Garantizar la disponibilidad y la gestión sostenible del agua y el saneamiento para todos; 7. Garantizar el acceso a una energía asequible, fiable, sostenible y moderna para todos; 9 . Construir infraestructuras resilientes, promover la industrialización inclusiva y sostenible y fomentar la innovación. 11. Lograr que las ciudades y los asentamientos humanos sean inclusivos, seguros, resilientes y sostenibles; y 13. Adoptar medidas urgentes para combatir el cambio climático y sus efectos.

De la Nueva Agenda Urbana de Hábitat III- NAU, se han considerado aspectos relacionados con el

\section{Compromisos de transformación en pro del desarrollo urbano sostenible.}

- El desarrollo urbano sostenible en pro de la inclusión social y la erradicación de la pobreza, en los puntos 29, 32, 34, 35, 36, 37 y 41.

- Prosperidad urbana sostenible e inclusiva y oportunidades para todos en los puntos 41,44 , $45,50,52,54$ y 62, y

- Desarrollo urbano resiliente y ambientalmente sostenible $63,64,65,66,67,68,69,70,72$, $73,74,75,76,77,78,79,80,97,100,101,104,107,108,109,110,111,112,113,114$ y 115.

En el apartado siguiente, se exponen los lineamientos de la Política de Gestión de Riesgos del Paraguay, a la que se agregan aspectos destacados de las normas internacionales de referencia.

\subsection{La Política de Gestión de Riesgos del Paraguay- PGR Py}

La PGR Py, aprobada por Decreto 1402/ 2014, deriva de la Ley 2615/2005, de creación de la Secretaría de Emergencia Nacional- SEN, para "prevenir y contrarrestar los efectos de las emergencias y los desastres originados por los agentes de la naturaleza o de cualquier otro origen..." (Ley2615, 2005: Pág. 1). Se basa también en los Objetivos del Milenio- ODM, y en las Prioridades de Acción del MAH. 
Este instrumento ubicado entre las tecnologías intangibles, tecnología de gestión, se estructura en 4 Pilares, cada uno de los cuales, a su vez, posee lineamientos de acción, que, contrastados con los instrumentos internacionales antes presentados, permiten evidenciar los aspectos que deberían ser fortalecidos o incorporados en relación con las Tecnologías del Hábitat e Infraestructuras. Tecnologías de Gestión. A continuación, y en virtud de la extensión posible del trabajo, en este apartado, se abordarán fundamentalmente las Tecnologías del Hábitat e infraestructuras involucradas en la Política de Gestión de Riesgos del país, relacionadas a las áreas urbanas, desde la perspectiva de la prevención de los riesgos (que implica actuar sobre las Amenazas y las Vulnerabilidades y aumentar las Capacidades) y no precisamente desde la gestión de los desastres, e igualmente de aquellas que pueden constituirse en aportes de la Universidad. A saber:

\section{Lineamientos Estratégicos}

\section{PILAR 1: Fortalecimiento de Capacidades Institucionales}

Lineamiento 1, 6. 11 y 12: Fortalecer, mejorar, potenciar la capacidad presupuestaria, estructural, legal operativa y de talentos humanos de la SEN en su rol como órgano rector articulador de las acciones relacionadas con la Gestión y Reducción de Riesgos de Desastres de la Republica. Diseñar un sistema de incentivos y estímulo para los actores y sectores de la sociedad que incorporen la gestión de riesgos como prioridad en el marco de sus acciones operativas y estratégicas y un sistema de sanciones administrativas, civiles y penales, para los actores y sectores que incumplan la normativa y las leyes relacionadas a la Gestión y Reducción de Riesgos de Desastres, así como para quienes provoquen situaciones de riesgo o desastres. Profesionalizar la gestión de las instituciones que integran el Sistema de Gestión y Reducción de Riesgos de Desastres y generar condiciones para la capacitación permanente de los distintos actores que componen el mismo.

A este respecto el MAH (2005), profundizado por SENDAI (2015) señala la importancia de desarrollar el, voluntariado, que, estructurado en el marco de la gestión de riesgos- GR, se avizora como potenciador de la eficiencia de las acciones del Sistema Nacional de Gestión y Reducción de Riesgos de Desastre- SNGRRD (Ej. en capacitación/educación y respuesta ante emergencias). No menos importante resulta fortalecer la capacidad normativa y técnica para la gestión (regional, nacional y local) de desastres, incluyendo capacidades en tecnología/materiales, y en formación de recursos humanos. Igualmente, para preparar y fortalecer enfoques regionales coordinados (crear o perfeccionar políticas, mecanismos operacionales, planes y sistemas de comunicación regionales) para la preparación y respuesta rápida y eficaz ante situaciones de desastre que rebasen la capacidad nacional.

Lineamiento 2, 3, 4 y 5: Instalar como prioridad nacional la temática de la Gestión y Reducción de Riesgos de Desastres en las políticas, planes, programas, proyectos y acciones desarrollados por las instituciones que lo componen. Crear e implementar el Sistema Nacional de Gestión y Reducción de Riesgos de Desastres- SNGRRD, con la SEN, como coordinadora de actores nacionales e internacionales con sus roles y acciones específicas. Definir la estructura organizacional, procedimientos, cargos, funciones y talentos humanos necesarios para la asignación y uso de los recursos propios de cada actor, así como de los asignados por el sistema, para cumplir con eficacia las funciones y mandamientos establecidos en esta política, así como...los planes estratégicos y operativos que puedan derivar de ella. 
Desde el planteamiento de los ODS 2030 y en relación con la problemática del acceso al agua y el saneamiento y disponibilidad de agua (sequías), como aspectos que generan riesgos vitales y de salud para la población, particularmente para las comunidades vulnerables, resulta fundamental, en el Paraguay, la articulación de varias instituciones (SEN, Municipalidades, SEAM, ESSAP, SENACSA y empresas privadas -aguateras).

Lineamiento 7 y 8: Elaborar e implementar procedimientos para el monitoreo, control y seguimiento de los planes, programas, proyectos y acciones previstos en el Sistema Nacional de Gestión y Reducción y Riesgos de Desastres. Igualmente elaborar e implementar procedimientos para la supervisión/control de ejecución y uso de recursos y rendición de cuentas.

Relacionado con este lineamiento el $\mathrm{MAH}$, recomienda preparar y revisar o actualizar regularmente las políticas y planes de preparación y contingencia en todos los niveles, especialmente para zonas y grupos vulnerables y para proteger y restablecer los ecosistemas relacionados con el agua (humedales, los ríos, los acuíferos y lagos). También establecer, mejorar y fomentar instrumentos de vigilancia para la reducción del riesgo de desastre en el contexto de la política y la planificación del uso de la tierra y para reducir el riesgo y los factores de vulnerabilidad, mediante la gestión integrada del medio ambiente y los recursos naturales y la elaboración de medidas estructurales y no estructurales (Ej. control integrado de las inundaciones o la gestión adecuada de los ecosistemas frágiles) En general estos postulados son también sostenidos por SENDAI (2015).

Lineamiento 9: Impulsar el mejoramiento permanente de

- la infraestructura edilicia con criterios de diseño universal y accesibilidad.

Para el MAH 2005, en los procesos planificación urbana y en los proyectos de infraestructuras importantes, deben considerarse los riesgos de desastre y la evaluación de su repercusión social, económica y ambiental, relacionados a los criterios de diseño, aprobación y ejecución. Recomienda proteger y mejorar las instalaciones públicas e infraestructuras de importancia clave (Ej. Instalaciones de atención primaria de salud. Que los nuevos hospitales puedan seguir funcionando en medio de desastres, con diseño adecuado, retro adaptación, reedificación, con refuerzos para que resistan a las amenazas y como medidas de mitigación de los riesgos.

Los programas de mejoramiento de barrios, la vivienda precaria y su ubicación en zonas de alto riesgo, deben tener atención prioritaria, y así mismo, evitar que los programas para desplazados aumenten el riesgo y la vulnerabilidad ante las amenazas, considerando medidas de reducción de los riesgos de desastre a largo plazo, en los procesos de recuperación y rehabilitación de desastres. La NAU, plantea así mismo infraestructura y viviendas adecuadas asequibles y resilientes y apoya la producción social del Hábitat, mientras aboga también por construcciones energéticamente eficientes, seguras, bajando las emisiones de gases de efecto invernadero en los materiales locales.

Reglamentos y normas de construcción u otros instrumentos a nivel nacional o local y también las prácticas de rehabilitación y reconstrucción deben revisarse con miras propiciar la construcción de estructuras resistentes a los desastres, facilitar su aplicación (particularmente en los asentamientos humanos precarios), reforzando la capacidad de vigilar y hacer cumplir dichos reglamentos. Se promueve, el estímulo a la cultura de prevención de los desastres en el sector privado: que las empresas de construcción contemplen medidas de reducción de riesgos. (MAH, 2005; SENDAI, 2015) 
- equipos para alerta temprana, instrumental y logística con lo s ajustes razonables necesarios, en las instituciones que componen el sistema para la prevención y respuesta en situaciones de emergencias y desastres.

De hecho, el intercambio de información y coordinación respecto de la alerta temprana, la reducción del riesgo de desastre, la respuesta a las situaciones de desastre, el desarrollo y otras actividades entre instituciones importa, en la perspectiva de la integralidad de la reducción del riesgo de desastre.

\section{PILAR 2: Financiamiento}

Este Pilar y sus Lineamientos, no fueron desarrollados por constituir aspectos fuera del marco de tratamientos señalado al principio del apartado.

\section{PILAR 3: Educación, Comunicación y Participación Ciudadana}

Lineamiento I, 2, 3, 4: Incorporar la Gestión y Reducción de Riegos de Desastres a las mallas curriculares de todos los niveles educativos formales (inicial, básica, media, superior no universitaria y universitaria) del país. Revisar y actualizar los planes e iniciativas educativas existentes en la Gestión y Reducción de Riesgos de Desastres (entre ellos el Plan Nacional de Gestión de Riesgos para el sector Educativo-PNEGER) y aquellas afines o transversales, arbitrar así mismo las medidas conducentes a la debida implementación del PNEGER para la educación inicial, básica y media, a lo cual el Ministerio de educación se halla abocado. Capitalizar las capacidades de los Gobiernos Central, Departamental y Municipal; de los sectores y actores no gubernamentales de la sociedad organizada en particular y de la sociedad toda en general, tanto local como internacional como vehículo de educación no formal e informal para la Gestión y Reducción de Riesgos de Desastres.

Este Pilar y sus lineamientos interesan particularmente a la Universidad, como centro de construcción y de transferencia de conocimientos a la sociedad. EI MAH refiere en este sentido, a la implementación de programas (evaluación de riesgos, reducción de los efectos de las amenazas y preparación para desastres) en las escuelas, así como programas de formación y enseñanza de la gestión y la reducción de los riesgos de desastre para planificadores del desarrollo urbano, administradores de situaciones de emergencia, funcionarios municipales, etc., en las instituciones de enseñanza superior y al mismo tiempo promover también la formación comunitaria.

Lineamiento 8: Crear, fortalecer y potenciar las instancias, espacios, estructuras, canales y capacidades institucionales que permitan fomentar y potenciar la participación ciudadana y en particular las personas con discapacidad y las organizaciones que las representan en el diagnóstico, diseño, implementación, ejecución, control, seguimiento y rendición de cuentas de las Políticas Públicas y demás planes, programas y proyectos que surjan de ella en el ámbito de la Gestión y Reducción de Riesgo.

En la perspectiva del MAH, 2005 y de los ODS 2030, vale promover la creación del voluntariado, por ej. en las unidades académicas que imparten cátedras relacionadas a los riesgos urbanos (FADA UNA), que podrían activarse desde las actividades de Extensión universitaria (créditos). También apoyar y fortalecer la participación de las comunidades locales en la mejora de la gestión del agua y el saneamiento. 


\section{PILAR 4: Gestión del Conocimiento y Tecnología.}

Respecto de éste Pilar, interesan los siguientes lineamientos:

Lineamiento 7, 4, 6: Crear un sistema de información que recopile, genere, centralice, administre, sistematice y provea la información histórica y actual, así mismo crear y mantener actualizados acervos (conocimientos empíricos, experiencias de la Comunidad) y conocimientos ciertos, así como la transferencia de estos y procesos de innovación correspondientes, en el marco del Sistema de Gestión y Reducción de Riesgos de Desastres, para la producción de tecnología e información conducente a reducir y/o eliminar amenazas, vulnerabilidades y riesgos, y que sirva de base para la ejecución de planes y medidas de Gestión y Reducción de Riesgos de Desastres.

EI MAH, encuentra necesaria la creación y mejora de las bases de datos y promoción del intercambio y la divulgación de datos para la evaluación, la vigilancia y la alerta temprana a nivel internacional, regional, nacional y local. Así mismo, incluir la evaluación del riesgo de desastre en la planificación urbana, en zonas montañosas y en llanuras costeras inundables, identificando zonas disponibles y seguras para los asentamientos humanos. Esto resulta particularmente significativo en Asunción, que constituye el Municipio con la mayor problemática del país, relacionada a damnificados y desplazados por causa de las crecidas del Río Paraguay desde el 2014 hasta estos días y que en cada evento no logra resolver adecuadamente los espacios para albergues provisorios.

Tanto el MAH (2005), SENDAI 2015, como los ODS (2030), encuentran muy necesario profundizar el conocimiento de las amenazas y su evolución, así como de los factores físicos, sociales, económicos y ambientales de vulnerabilidad (a corto y largo plazo). Determinar estadísticas y datos sobre los riesgos y de los efectos (impacto, pérdidas que ocasionan) de los desastres, que comprueben la existencia de patrones/tendencias del riesgo y permitan la adopción de las medidas oportunas correspondientes. Contemplar también los posibles riesgos de desastres relacionados con el cambio de las condiciones sociales, económicas, ambientales y de uso de la tierra, y las consecuencias de las amenazas naturales y antrópicas generadoras de éstos. Incluir la evaluación de los riesgos de desastre en los planes de urbanismo y la gestión de los asentamientos humanos expuestos a desastres, en particular las zonas densamente pobladas y los asentamientos en rápida urbanización. Integrar a este proceso la identificación y la información de riesgos asociados al clima actual y futuro y su reducción posible. A dicho efecto, resultan indispensables los Mapas de riesgos, la construcción sistemas de indicadores para controlar los riesgos y la vulnerabilidad nacional y subnacional. También, los estudios, análisis e informes sobre los cambios a largo plazo y las cuestiones emergentes que pueden aumentar la vulnerabilidad y los riesgos o afectar la capacidad, como base de evaluaciones periódicas de los riesgos a nivel nacional y local, cuyos resultados deberían difundirse tanto para las autoridades responsables, como para las comunidades expuestas y la ciudadanía en general. Se observa la conveniencia de que las instituciones a cargo del desarrollo urbano provean información a los interesados, sobre los riesgos posibles y así mismo sobre las posibilidades de reducción de estos, antes del inicio de proyectos de construcción o de la compra/venta de tierras.

También importan los ejercicios periódicos de preparación para casos de desastre, incluidos ejercicios de evacuación, a fin de alcanzar respuestas rápidas y eficaces ante situaciones de desastre y el acceso a los suministros esenciales de socorro alimentario y de otro tipo con arreglo a las necesidades locales (MAH, 2005) 
Lineamiento 5, 8: Promover el intercambio y transferencia de conocimientos, experiencias y tecnologías en materia de Gestión y Reducción de Riesgos de Desastres entre el Paraguay y otros países y Organizaciones Internacionales, Regionales y Sub- Regionales. Fomentar los mecanismos que impulsen el uso y la transferencia de tecnología y conocimientos relacionados a la Gestión y Reducción de Riesgos de Desastres desde sus fuentes hacia el Sistema Nacional de Gestión y Reducción de Riesgos de Desastres y su ente rector (SEN).

La Cooperación internacional será importante para intercambiar información, evaluar y vigilar los riesgos transfronterizos (regionales y emergentes) por ejemplo en el caso de las cuencas fluviales y para la emisión de alertas tempranas.

EI MAH (2005), recomienda también promover "uso, aplicación y asequibilidad de las últimas tecnologías de la información y la comunicación y las tecnologías espaciales y los servicios conexos, así como las observaciones terrestres, para contribuir a la reducción del riesgo de desastre...".

Lineamiento 9, 3: Fomentar la investigación conducente a ampliar la base de conocimiento y tecnología relacionada a la Gestión y Reducción de Riesgos de Desastres. Promover la creatividad y las condiciones para desarrollar la innovación con nuevos abordajes, métodos, soluciones y nuevas tecnologías para la reducción de amenazas, vulnerabilidades y riesgos.

Estos lineamientos, interesan particularmente a la Universidad. De acuerdo con los postulados del $\mathrm{MAH}$, es necesaria la creación y mantenimiento de infraestructuras y capacidades científicas, tecnológicas, técnicas e institucionales necesarias para estudiar, observar, analizar, cartografiar y pronosticar las amenazas, los factores de vulnerabilidad y los efectos de los desastres. Así mismo importa promover el uso de los sistemas de información geográfica, el modelado y la predicción de las amenazas, el modelado y pronóstico meteorológico y climático, y en particular mejorar las capacidades de vigilancia y evaluación regionales"

Se requiere el fortalecimiento de capacidades técnicas y científicas para elaborar y aplicar metodologías, estudios y modelos de evaluación de los factores de vulnerabilidad ante las amenazas de origen geológico, meteorológico, hidrológico-climático y sus efectos. Derivados de estos procesos de investigación, desarrollar servicios de información sobre tecnologías de reducción del riesgo de desastre rentables y fáciles de aplicar, buenas prácticas y lecciones aprendidas sobre las políticas, los planes y medidas de reducción del riesgo de desastre. Fortalecer los espacios de registros y análisis: estadísticas, representaciones cartográficas de las amenazas, riesgos y efectos de los desastres y desarrollo de metodologías comunes de evaluación y vigilancia de los riesgos. Incorporar las evaluaciones de riesgos múltiples y los análisis de costo-beneficio de las medidas de reducción de los riesgos, a los procesos de decisión regional, nacional y local. (MAH, 2005).

Actualizar y divulgar ampliamente una terminología internacional normalizada sobre la reducción del riesgo de desastre, para la investigación, los programas de formación y los programas de información pública

Investigación para los sistemas de Alerta temprana que permitan advertir en tiempo y forma clara a las personas en riesgo, sobre el fenómeno a ocurrir y sobre las acciones que se deben realizar para contribuir a la eficacia de los operativos de gestión de desastre. Los sistemas de alerta temprana deben probarse y evaluarse periódicamente. (MAH, 2005) 
SENDAI (2015), profundiza los postulados del MAH (2005) planteando la necesidad de desarrollar investigaciones relacionadas al acceso al agua, a servicios de saneamiento e higiene adecuados y a la reducción de la contaminación (eliminación/reducción de vertidos y emisión de productos químicos y materiales peligrosos), para reducir significativamente las aguas residuales sin tratar, aumentar el reciclado y la reutilización sin riesgo del agua.

Los ODS 2030, también desarrollan señalamientos en relación con el manejo de los recursos hídricos, promoviendo el uso eficiente de estos en todos los sectores. Ello implica asegurar la sostenibilidad de la disponibilidad y extracción de agua dulce, haciendo frente a la escasez del vital líquido, al tiempo que se reduce la población sin acceso al mismo, implementando la gestión integrada de los recursos hídricos incluso en niveles transfronterizos. A dicho efecto, crear capacidades por medio de actividades y programas relativos al agua y el saneamiento: captación de agua, desalinización, uso eficiente de los recursos hídricos, tratamiento de aguas residuales, reciclado y tecnologías de reutilización, etc. En este sentido la investigación FADA UNA, mencionada en los inicios de este trabajo, ha expuesto aspectos que afectan las condiciones de los cauces hídricos urbanos, entre ellas los procesos de ocupación actual del territorio para el uso habitacional, en las ciudades del país y los asentamientos (precarios o no) en sus bordes, lo cual requiere estudios sobre la capacidad portante de los suelos en los bordes, o para mitigar y/o eliminar otros riesgos que se desarrollan en la actualidad y de ser posible capitalizar las obras de protección ya realizadas. Se observa la urgente necesidad de atender los cauces hídricos, en los procesos de antropización del territorio, tanto para prevenir y/o eliminar o cuando menos mitigar las situaciones de riesgo de los Asentamientos Precarios Urbanos, o de los Asentamientos formales, como para la preservación de estos como valiosísimo recurso natural.

Los ODS 2030, refieren a la infraestructura resiliente, la industrialización inclusiva y sostenible (reconversión, modernizar la infraestructura, adopción de tecnologías y procesos industriales limpios y ambientalmente racionales), al fomento de la innovación, a la inversión en infraestructura (transporte, riego, energía y tecnología de la información y las comunicaciones) más resistentes al cambio climático enfatizando el acceso asequible y equitativo para todos y la de las industrias para que sean sostenibles (uso de recursos más eficaces). Aumentar la investigación científica y mejorar la capacidad tecnológica de los sectores industriales. Desarrollar tecnología e innovación que garantice el marco normativo propicio para la diversificación industrial.

\section{CONCLUSIONES}

Se ha visto a partir de la exposición precedente, que, frente a la concentración de la población en ciudades, a la urbanización rápida, y todos los problemas que acarrean, la Gestión de Riesgos constituye una herramienta fundamental en la perspectiva del desarrollo sostenible de un país, y en vista de su amplia influencia en diferentes ámbitos, requiere el desarrollo de Tecnologías de Gestión relacionadas con el Hábitat urbano y así mismo con las infraestructuras que lo componen.

Si bien la PGR Py, está planteada en los términos amplios de los lineamientos, este trabajo deja entrever lo mucho que implica cada uno de ellos, y lo mucho que hay que hacer, desde el conocimiento de los Riesgos y sus componentes (Amenazas, Vulnerabilidades y Capacidades), hasta tomar las medidas preventivas necesarias, pasando por las emergencias y posterior recuperación, rehabilitación y reconstrucción. Queda claro así mismo, que, en muchos casos, la participación de la Universidad en el proceso puede llegar a ser estratégica, desde la construcción 
de conocimientos por medio de la investigación, el apoyo a la creación de acervos y bases de datos, así como desde la dirección de emprendimientos y servicios realizados para la prevención de los riesgos o para la elaboración/ implementación de medidas estructurales y no estructurales, destinadas a la mitigación y/o eliminación de los riesgos de diversa índole.

Se ha visto también en el contraste de la PGR Py, con la normativa internacional, que existen aspectos que no están presentes en la misma o que deben ser fortalecidos, a la luz de los riesgos y eventos de desastre cuyos efectos se han intensificado en el país. En este contexto, se avizora como oportunidad cierta, la participación de la Universidad, como agente de producción de conocimiento tanto de las amenazas y vulnerabilidades y los riesgos, como también en lo atinente a la planificación urbana y el ordenamiento territorial, y también como coadyuvante en los procesos de creación de acervos y bases de datos sobre las Amenazas, así como las condiciones de exposición de las Comunidades a sus efectos. De hecho y particularmente la FADA UNA, puede participar en buena parte de los procesos preventivos de la GR y se observa que esta institución puede aportar de diversas maneras: A partir del desarrollo de Trabajos Finales de Grado de las modalidades Investigación o Pasantía, desde la propia Extensión universitaria, o desde la Cátedra de Riesgos Urbanos, impulsando por ejemplo la creación de un Voluntariado formado académicamente, o también desde los Trabajos realizados por los Docentes Investigadores de Dedicación Completa- DIDCom, que han abierto una líneas de trabajo de investigación, así como un amplio espectro de cuestiones que existen por investigar en el país. Entre ellas, abordar, por ejemplo, la problemática de los desplazamientos generados por los desbordes de ríos y arroyos, que además de salvar la emergencia, requiere un abordaje comprometido de las condiciones extremas que se viven en los refugios transitorios durante las aguas altas. Así mismo, generar acciones de control sanitario antes de que los afectados vuelvan a sus lugares de origen.

\section{BIBLIOGRAFÍA}

Decreto 1402 (2014) Por el Cual se aprueba el Documento sobre Política Nacional de Gestión y Reducción de Riesgos (PNGR).

Ley 2615 (2005) que crea la Secretaría de Emergencia Nacional- SEN.

MAH (2010) Marco de Acción de Hyogo para 2005-2015. Aumento de la resiliencia de las naciones y las comunidades ante los desastres. Revisión de Medio término 2010-2011. (Provisional Translation).

ONU- EIRD- MAH (2005) Marco de Acción de Hyogo para 2005-2015. Aumento de la resiliencia de las naciones y las comunidades ante los desastres. Conferencia Mundial sobre la Reducción de los desastres.18 a 22 de enero de 2005, Kobe, Hyogo, Japón. Organización de las Naciones Unidas Estrategia Internacional de Reducción de Desastres. Kobe, Hyogo, Japón.

ONU (2015) Resolución aprobada por la Asamblea General el 25 de septiembre de 2015. Transformar nuestro mundo: la Agenda 2030 para el Desarrollo Sostenible.

ONU (2017) Nueva Agenda Urbana H III. Secretaría de Hábitat III. Impresión apoyo del Gobierno del Ecuador. ISBN: 978-92-1-132736-6

ONU - CEPAL (2018) Agenda 2030 y los Objetivos de Desarrollo Sostenible. Una oportunidad para América Latina y el Caribe. Publicación de las Naciones Unidas. Naciones Unidas, Santiago. 
Zoido, F.; De la Vega, S.; Piñeiro, A.; Morales G.; Mas, R.; Lois, RC.; González, JM. (2013) Diccionario de Urbanismo Geografía Urbana y de Ordenación del Territorio. $1^{a}$ Edición. Madrid (España). Ediciones Cátedra (Grupo Anaya, S.A.)

\section{Internet}

Arango Escobar, E (1986) Tecnologías del Hábitat. Material Presentado en el $13^{\circ}$. $14^{\circ}$. Taller Latinoamericano del CEHAP-PEVAL en 1986. http://www.bdigital.unal.edu.co/3047/1/GAETECNOLOGIAS.PDF - visitado abril, 2018)

González Escobar, LF (2001) La concepción Tecnológica del Hábitat. En Ensayos FORHUM 19Miradas al hábitat. Trabajo presentado en el Seminario Teórico de la Maestría en Hábitat de la Escuela del Hábitat-Cehap (segundo semestre del año 2001)

http://bdigital.unal.edu.co/2230/1/lfg02-For19.PDF - visitado abril, 2018)

Naciones Unidas (2015) Marco Sendai para la reducción del Riesgo de Desastres 2015-2030. https://www.unisdr.org/files/43291_spanishsendaiframeworkfordisasterri.pdf. (visitado,

Hábitat III (2015) Temas Hábitat III. 18 - Infraestructura Urbana y servicios básicos. Nueva York. http://habitat3.org/wp-content/uploads/Issue-Paper-18_Infraestructura_Urbana-SP.pdf 